\title{
Muscle Fatigue Assessment through Electrodermal Activity Analysis during Isometric Contraction
}

\author{
Alberto Greco*, Andrea Guidi, Federica Felici, Andrea Leo, Emiliano Ricciardi, \\ Matteo Bianchi, Antonio Bicchi, Luca Citi, Gaetano Valenza ${ }^{\ddagger}$ and Enzo Pasquale Scilingo ${ }^{\ddagger}$
}

\begin{abstract}
We studied the effects of muscle fatigue on the Autonomic Nervous System (ANS) dynamics. Specifically, we monitored the electrodermal activity (EDA) on 32 healthy subjects performing isometric biceps contraction. As assessed by means of an electromyography (EMG) analysis, 15 subjects showed muscle fatigue and 17 did not.

EDA signals were analyzed using the recently proposed cvxEDA model in order to decompose them into their phasic and tonic components and extract effective features to study ANS dynamics. A statistical comparison between the two groups of subjects was performed. Results revealed that relevant phasic EDA features significantly increased in the fatigued group. Moreover, a pattern recognition system was applied to the EDA dataset in order to automatically discriminate between fatigued and non-fatigued subjects. The proposed leave-one-subject-out KNN classifier showed an accuracy of $75.69 \%$. These results suggest the use of EDA as correlate of muscle fatigue, providing integrative information to the standard indices extracted from the EMG signals.
\end{abstract}

\section{INTRODUCTION}

Fatigue is a complex and widely used term, which can be related to both physiological and psychological states of a human [1], [2]. Muscle fatigue is defined as any exerciseinduced reduction in the ability of a muscle to generate force or power [3]. Indeed, it is not the point of task failure or the moment when the muscles become exhausted [4]. Rather, muscle fatigue is a decrease in the maximal force or power produced by muscle, and it gradually develops as soon as the sustained physical activity begins.

Muscle fatigue can depend upon many central and peripheral phenomena such as a motor deficit, a perception or a decline in mental function, or a gradual decrease in the force capacity of muscle [4]. All these phenomena involve different physiological mechanisms. Accordingly, there is no single cause of muscle fatigue [5], rather it involves multiple physiological, electrical and emotional processes. Several indices of muscular fatigue have been proposed in the literature. Particularly, the analysis in the time-frequency domain of the surface electromyography (sEMG) is a simple but reliable method to estimate the muscular fatigue in

The research leading to these results has received partial funding from the EU H2020 project "SoftPro: Synergy-based Open-source Foundations and Technologies for Prosthetics and RehabilitatiOn" (H2020-ICT-688857).

A.G., A.G., M.B., G.V., E.P.S. are with the department of Information Engineering and Research Center "E. Piaggio", School of Engineering, University of Pisa, Via G. Caruso 16 - 56122, Pisa, Italy.

F.F and A.B. are with the Advanced Robotics Department, Istituto Italiano di Tecnologia (IIT), Genova, Italy

A.L. and E.R. are with the IMT School for Advanced Studies Lucca, Piazza San Ponziano, 6, I-55100 Lucca, Italy

L.C. is with the School of Computer Science and Electronic Engineering, University of Essex, Colchester CO4 3SQ, UK.

‡ Senior Authors; * Corresponding author different fields of study and is considered a robust alternative to the gold standard approach of the intramuscular EMG. [6]-[8].

Several studies have shown that during voluntary muscle contractions the autonomic nervous system (ANS) is activated and modulated by two different mechanisms of neural control [9], [10]. More specifically, both the sympathetic and parasympathetic branches of the ANS are modulated by the brain activity within the brainstem, also called central command [9], [11], [12], and reflex activity primarily involving inputs from chemo-, mechano- and baroreceptor afferents [13], [14]. The effects of ANS activation are evident on the heart-rate variability, respiration rate and blood pressure responses to exercise and fatigue [15], [16], [16]-[18]. An increasing heart rate, as well as oxygen uptake, has been observed during prolonged exercises. Prior art reports also a strong correlation between ANS and electrodermal activity (EDA) phenomena [19]-[22]. More specifically, EDA is directly controlled by the sympathetic branch of the ANS [23]. The EDA is a widely used physiological signal that reflects the output of integrated attentional and affective and motivational processes. EDA is used as a biomarker of individual characteristics of emotional responsiveness [23] [26], as an index for direct examination of stress-related effects and mental fatigue. Contrary to the intramuscular EMG, EDA is not invasive and also much easier to be acquired through superficial electrode.

However, to our knowledge it has not been studied yet as a correlate of muscle fatigue induced by physical exercise.

Therefore, the aim of this study is to examine whether parameters extracted from the EDA during isometric exercise inducing muscular fatigue might be able to statistically discriminate a group of fatigued subjects from a group of control subjects. Moreover, we propose a pattern recognition system able to automatically discriminate muscular fatigue using features extracted exclusively from the EDA signals.

\section{METHODS}

\section{A. Subject Recruitment and Experimental protocol}

Thirty-two subjects, aged $29.25 \pm 3.38$ (16 males), signed an informed consent to take part in the study. All the subjects were right-handers and did not report any physical or mental disease.

We designed an experiment in order to monitor EDA during a muscle fatigue task. The experimental protocol (Fig. 1) was divided into three stages. In the first stage, subjects were asked to maximally contract the biceps of the right arm holding a dynamometer in order to measure their maximum voluntary isometric contraction (MVC) [5], 


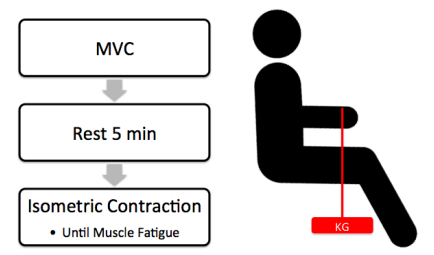

Fig. 1. Experimental protocol scheme

[27]. We performed three MVC measures and computed their average. Electrodermal sensors were placed on the distal phalanxes of the index and middle fingers of the left hand. Likewise, the surface EMG electrodes were positioned on the right biceps.

The second stage consisted of a 5-minute resting phase, during which subject relaxed and the EDA and sEMG started to be recorded.

In the third session, a load equal to $40 \%$ of MVC was connected to the right wrist of the subject. Then, each subject was asked to perform an isometric exercise lifting the arm to a horizontal position and maintaining it until it became impossible to maintain the horizontal position. During the exercise both EDA and biceps-related sEMG were recorded using the Biopac MP150 system (with a sampling frequency of $2 \mathrm{kHz}$ ).

\section{B. sEMG Analysis}

Surface EMG analysis was used as reference analysis in order to distinguish fatigued subjects from non-fatigued subjects [28]. EMG spectral analysis was performed using the Discrete Fast Fourier Transform to estimate the power spectra in a $1 \mathrm{~s}$ moving window, without overlap. For each time window, we calculated the mean frequency value in order to have the progression of the Mean Frequency (MF) signal in time. Following the current literature, subjects who showed a monotonic decrease of the MF signal were considered as fatigued [28], [29].

\section{EDA Processing using cvxEDA algorithm}

EDA refers to change in the electrical properties of the skin that are directly related to the eccrine sweat gland activity. The EDA is measured monitoring skin conductance (SC) changes on specific sites of the body where the concentration of the eccrine glands is high. More specifically, in this study the EDA was acquired on the finger phalanxes of the nondominant hand.

Due to the direct control of the sweat glands activity performed by the sympathetic branch of the ANS (and in particular the sudomotor nerve), the EDA analysis is considered one of the best way to monitor the sudomotor nerve activity (SMNA) and, consequently, the ANS activity.

We can decompose the EDA signals in two components, phasic and tonic, which differ for their time scales and their relationships with the external stimuli [23], [30]. More in detail, the tonic component represents the conductance level (SCL) of the EDA signal, i.e., the slow-varying baseline level. The phasic signal is instead a faster variation in the EDA directly related to an external stimulus. A single response to a stimulus is generally called skin conductance response (SCR).
The whole EDA signal results from the sum of these components, that provide relevant and non-redundant information about the ANS dynamics. We extracted these information by decomposing the EDA signal with an approach formerly validated: the cvxEDA model [30] (available online at:

http://www.mathworks.com/matlabcentral/fileexchange/53326cvxeda).

Here we briefly summarize the main methodological steps formulating the cvxEDA model of EDA dynamics (details can be found in [30]). The model assumes EDA as the output of a linear time-invariant system to a sparse nonnegative driver signal. CvxEDA described the EDA signal as a sum of three terms: a phasic component (r), a smooth tonic component $(\mathrm{t})$, and an additive independent and identically distributed zero-average Gaussian noise term $\epsilon: y=r+t+\epsilon$. The tonic component is modeled by the sum of an offset term, a linear trend and a smooth signal given by means of a cubic spline in accordance to the physiological characteristics: $t=B \ell+C d$. The phasic component is the result of a convolution between a signal that represents the SMNA, $p$, and an impulse response shaped like a biexponential Bateman function [31] and modeled by an autoregressive moving average model, $A^{-1} M$.

Using an auxiliary variable $q$ such that $q=A^{-1} p$ and $r=M q$. The final observation model results as

$$
y=M q+B \ell+C d+\epsilon .
$$

Given the EDA model (1), we aim at identifying the tonic component $(t)$ and the maximum a posteriori (MAP) neural driver SMNA $(p)$, parametrized by $[q, \ell, d]$, for the observed EDA signal $(y)$. CvxEDA rewrites the MAP problem as a constrained minimization QP convex problem:

$$
\begin{aligned}
\operatorname{minimize} & \frac{1}{2}\|M q+B \ell+C d-y\|_{2}^{2}+\alpha \delta\|A q\|_{1}+\frac{\gamma}{2}\|\ell\|_{2}^{2} \\
\text { subj. to } & A q \geq 0 .
\end{aligned}
$$

Where $\delta$ is the sampling time. CvxEDA finds the optimal $[q, \ell, d]$ and consequently the optimal tonic and phasic components that include the prior knowledge about the spiking sparse nature and nonnegativity of the neural signal representing the SMNA $(p)$ and the smoothness of the tonic SCL.

TABLE I

LIST OF FEATURES EXTRACTED FROM PHASIC AND TONIC EDA.

\begin{tabular}{|c|c|}
\hline Feature & Description \\
\hline SCRfreq & frequency of significant SCR wrw \\
\hline AUCphasic & Area under the curve of phasic component wrw $(\mu S s)$ \\
\hline MaxPeak & maximum amplitude of significant peaks of \\
& SMNA signal wrw ${ }^{1}(\mu S)$ \\
\hline MeanAMP & Mean value of the SMNA signal wrw $(\mu S)$ \\
\hline STDphasic & Standard deviation of SMNA signal wrw $(\mu S$ \\
\hline STDtonic & Standard deviation of tonic signal wrw $(\mu S$ \\
\hline MeanTonic & Mean value of the tonic component wrw $(\mu S)$ \\
\hline EDAsymp & $\begin{array}{c}\text { Index of sympathetic nervous system activity }\left(\mu S^{2}\right) . \\
\text { PSD of the EDA signal within the } 0.045-0.25 \mathrm{~Hz} \text { band. }\end{array}$ \\
\hline \multicolumn{2}{|c|}{ wrw = within response window (i.e., 5 secs before the end of the task) }
\end{tabular}

As last processing step, we extracted several features from both the tonic and phasic signal as summarized in Table I. In addition to the standard statistical features in the time 
domain, we computed also the power spectral density (PSD) of the EDA signal in the frequency band $0.045-0.25 \mathrm{~Hz}$ (i.e. EDAsymp), which has been demonstrated to be a reliable parameter for the assessment of the sympathetic nervous system activity [32].

\section{Statstical Analysis and Classification Procedure}

Standard EDA time-domain features, as well as frequency EDAsymp index were calculated for each subject of both groups (i.e. fatigued and non-fatigued). We performed two kinds of analysis: considering the whole session and only the last 5 seconds when the effort was maximum. For every feature, we compared values between fatigued and nonfatigued conditions using the non-parametric Mann-Withney $\mathrm{U}$ test.

In addition, we applied an automatic pattern recognition algorithm in order to distinguish between the two groups of subjects using EDA dynamics exclusively. The algorithm implemented a leave-one-subject-out procedure (LOSO) applied to a KNN-based classifier. Specifically, considering $N$ subjects, iteratively we split the feature set in a training set, comprised of the data of $(N-1)$ participants, and a test set with the data of the remaining subject. Moreover, within the LOSO scheme, at each iteration, we performed both a feature selection and an optimization of the KNN parameter (i.e., the number of neighbors, $k$ ). More specifically, the selected feature-set was achieved identifying among all combinations of the 8 features, the one performing the highest recognition accuracy within each training set (i.e., by means a further nested LOSO).

Results of the classification procedure will be shown in terms of confusion matrix. The diagonal of the $2 \times 2$ matrix represents the percentage subjects correctly classified for each of the two classes (true positives and true negatives). Instead, in the anti-diagonal, we showed the percentage of the fatigued subjects that were incorrectly marked as nonfatigued and vice versa.

\section{RESULTS}

Results of the sEMG time-frequency analysis demonstrated that 15 subjects suffered from muscle fatigue. Indeed, a monotonic decrease in time of the MF value were found. Instead, the remaining 17 subjects did not reach the muscular fatigue.

We then compared EDA features between the two groups. Considering EDA signals of the whole isometric contraction session, no statistical differences were found between the two groups. Instead, considering only the last 5 seconds of the isometric exercise, when muscle fatigue was more evident, we obtained the results summarized in Fig. 2. These statistical outcomes showed that the tonic features did not display significant differences. Instead, three relevant parameters of the phasic activity, i.e., the mean amplitude and the standard deviation and the maximum SCR, were significantly different between the two groups of subjects.

Result of the classification procedure is shown in Table II. The EDA features extracted using the cvxEDA model were able to recognize the two groups of subjects with an
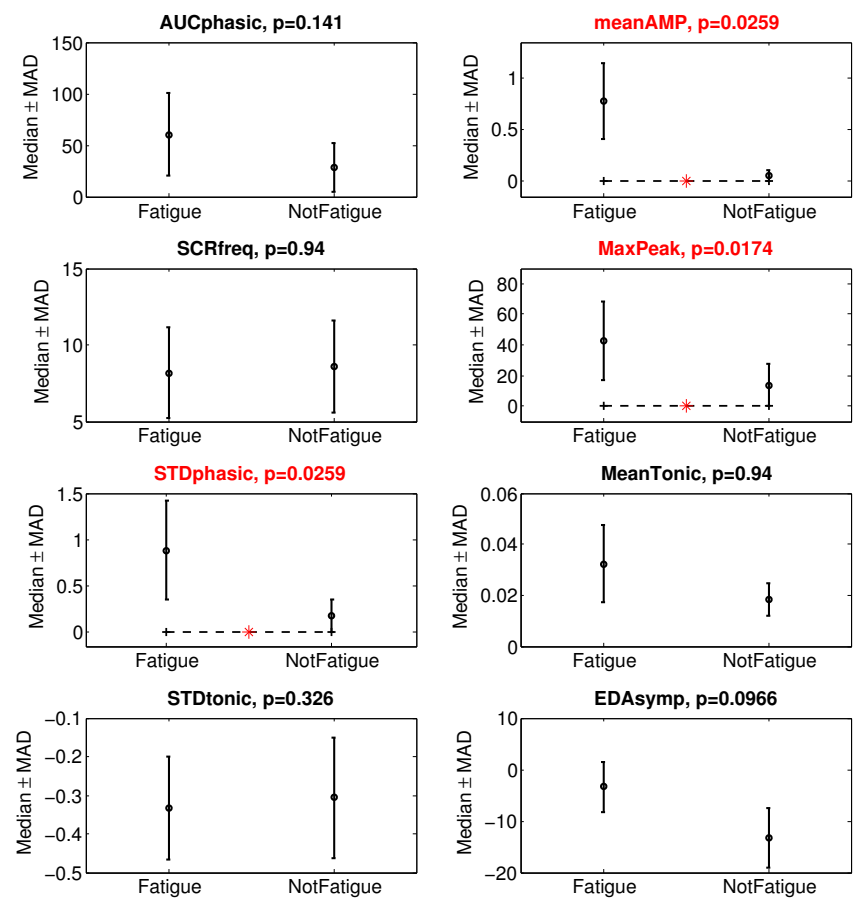

Fig. 2. Statistical comparison between fatigue group and non-fatigue group. Each figure corresponds to one of the EDA features.

TABLE II

CONFUSION MATRIX OF FATIGUED VS NON-FATIGUED GROUP USING CVXEDA FEATURES.

\begin{tabular}{|c|c|c|}
\hline KNN & NON-FATIGUE & FATIGUE \\
\hline NON-FATIGUE & $\mathbf{8 6 . 6 7 \%}$ & $35.39 \%$ \\
\hline FATIGUE & $13.33 \%$ & $\mathbf{6 4 . 7 1 \%}$ \\
\hline
\end{tabular}

average accuracy of $75.69 \%$. More specifically, the nonfatigued group was recognized with an accuracy of $86.67 \%$, whereas the fatigued group with a lower accuracy of $64.71 \%$.

\section{DISCUSSION AND CONCLUSION}

In this study, we investigated ANS correlates of muscle fatigue through the analysis of EDA. To this end, we monitored 32 healthy subjects (16 males) during a prolonged isometric contraction. The participants were divided in two groups, i.e., fatigued and non-fatigued, according to the sEMG frequency analysis, which is considered a reliable measurement for the detection of muscle fatigue.

We proposed both a statistical comparison and a pattern recognition analysis in order to distinguish the two groups of subjects using EDA signals. In order to extract effective features, the EDA signals was decomposed into its tonic and phasic components using the cvxEDA model [30].

Significant differences were found when considering the last 5 seconds of the isometric exercise. Considering the sympathetic control of the EDA phenomena [23], [33], this result was consistent with previous studies [27], [34], which claimed that the inhibition of the vagal tone was the predominant mechanism during the first part of an isometric exercise, whereas close to the completion of the exercise the sympathetic effector response started to prevail [27]. Accordingly, our findings showed that differences between the two groups did not involve the tonic component but the 
phasic one, which describes the fast response to external stimuli or motor activity [35]-[37].

In addition, we proposed a classifier able to recognize the fatigued and non-fatigued group of subjects using EDA features with an average accuracy as high as $75.69 \%$. Again, this indicated the possibility of using EDA as a musclefatigue correlate, alternatively to the sEMG.

In conclusion, our results suggest a strong relationships between muscle fatigue and phasic EDA, and indicate a significant increase of the sympathetic response in case of muscle fatigue. EDA could be a valid alternative to monitor the muscle fatigue, in fact, contrary to intramuscular EMG, EDA is non invasive and easy to be acquired also in ecological scenarios. Future works will aim to analyze possible gender differences, and to increase the accuracy of the pattern recognition system possibly by including features coming from other physiological signals (e.g. HRV, which adds the parasympathetic system information).

\section{REFERENCES}

[1] M. M. Bundele and R. Banerjee, "An svm classifier for fatiguedetection using skin conductance for use in the bits-lifeguard wearable computing system," in Emerging Trends in Engineering and Technology (ICETET), 2009 2nd International Conference on. IEEE, 2009, pp. 934-939.

[2] P. Bhat and A. Gupta, "A novel approach to detect localized muscle fatigue during isometric exercises," in Wearable and Implantable Body Sensor Networks (BSN), 2016 IEEE 13th International Conference on. IEEE, 2016, pp. 224-229.

[3] S. Gandevia, "Spinal and supraspinal factors in human muscle fatigue," Physiological reviews, vol. 81, no. 4, pp. 1725-1789, 2001.

[4] R. M. Enoka and J. Duchateau, "Muscle fatigue: what, why and how it influences muscle function," The Journal of physiology, vol. 586, no. 1, pp. 11-23, 2008.

[5] S. P. Cairns, A. J. Knicker, M. W. Thompson, and G. Sjøgaard, "Evaluation of models used to study neuromuscular fatigue," Exercise and sport sciences reviews, vol. 33, no. 1, pp. 9-16, 2005.

[6] K. Ito and Y. Hotta, "Surface electromyogram-based detection of muscle fatigue during cyclic dynamic contraction under blood flow restriction," in Engineering in Medicine and Biology Society (EMBC), 2014 36th Annual International Conference of the IEEE. IEEE, 2014, pp. 2205-2208.

[7] R. H. Chowdhury, M. B. Reaz, M. A. B. M. Ali, A. A. Bakar, K. Chellappan, and T. G. Chang, "Surface electromyography signal processing and classification techniques," Sensors, vol. 13, no. 9, pp. $12431-12466,2013$.

[8] D. MacIsaac, P. Parker, and K. Englehart, "A novel approach to localized muscle fatigue assessment," in Engineering in Medicine and Biology Society, 2003. Proceedings of the 25th Annual International Conference of the IEEE, vol. 3. IEEE, 2003, pp. 2487-2490.

[9] D. B. Friedman, C. Peel, and J. H. Mitchell, "Cardiovascular responses to voluntary and nonvoluntary static exercise in humans," Journal of Applied Physiology, vol. 73, no. 5, pp. 1982-1985, 1992.

[10] J. Mitchell, D. Reeves, H. Rogers, and N. Secher, "Epidural anaesthesia and cardiovascular responses to static exercise in man." The Journal of Physiology, vol. 417, no. 1, pp. 13-24, 1989.

[11] G. Goodwin, D. McCloskey, and J. Mitchell, "Cardiovascular and respiratory responses to changes in central command during isometric exercise at constant muscle tension," The Journal of Physiology, vol. 226, no. 1, pp. 173-190, 1972.

[12] F. Iellamo, R. Hughson, F. Castrucci, J. Legramante, G. Raimondi, G. Peruzzi, and G. Tallarida, "Evaluation of spontaneous baroreflex modulation of sinus node during isometric exercise in healthy humans," American Journal of Physiology-Heart and Circulatory Physiology, vol. 267, no. 3, pp. H994-H1001, 1994.

[13] L. B. Rowell and D. S. O'Leary, "Reflex control of the circulation during exercise: chemoreflexes and mechanoreflexes," Journal of Applied Physiology, vol. 69, no. 2, pp. 407-418, 1990.

[14] F. Iellamo, J. Legramante, G. Raimondi, and G. Peruzzi, "Baroreflex control of sinus node during dynamic exercise in humans: effects of central command and muscle reflexes," American Journal of Physiology-Heart and Circulatory Physiology, vol. 272, no. 3, pp. H1157-H1164, 1997.
[15] L. Sheldahl, L. Wann, P. S. Clifford, F. Tristani, L. Wolf, and J. Kalbfleisch, "Effect of central hypervolemia on cardiac performance during exercise," Journal of Applied Physiology, vol. 57, no. 6, pp. 1662-1667, 1984.

[16] J. Mitchell, F. Payne, B. Saltin, and B. Schibye, "The role of muscle mass in the cardiovascular response to static contractions," The Journal of Physiology, vol. 309, p. 45, 1980

[17] A. S. Leicht, W. H. Sinclair, and W. L. Spinks, "Effect of exercise mode on heart rate variability during steady state exercise," European journal of applied physiology, vol. 102, no. 2, pp. 195-204, 2008.

[18] M. Weippert, M. Behrens, R. Gonschorek, S. Bruhn, and K. Behrens, "Muscular contraction mode differently affects autonomic control during heart rate matched exercise," Frontiers in physiology, vol. 6, 2015.

[19] R. Calvo and S. D'Mello, "Affect detection: An interdisciplinary review of models, methods, and their applications," Affective Computing, IEEE Transactions on, vol. 1, no. 1, pp. 18-37, 2010.

[20] A. Lanatà, G. Valenza, A. Greco, C. Gentili, R. Bartolozzi, F. Bucchi, F. Frendo, and E. P. Scilingo, "How the autonomic nervous system and driving style change with incremental stressing conditions during simulated driving," IEEE Transactions on Intelligent Transportation Systems, vol. 16, no. 3, pp. 1505-1517, 2015.

[21] A. Greco, A. Lanata, L. Citi, N. Vanello, G. Valenza, and E. P. Scilingo, "Skin admittance measurement for emotion recognition: A study over frequency sweep," Electronics, vol. 5, no. 3, p. 46, 2016.

[22] A. Greco, G. Valenza, M. Nardelli, M. Bianchi, L. Citi, and E. P. Scilingo, "Force-velocity assessment of caress-like stimuli through the electrodermal activity processing: Advantages of a convex optimization approach," IEEE Transactions on Human-Machine Systems, vol. 47 , no. 1, pp. 91-100, 2017

[23] W. Boucsein, Electrodermal activity. Springer, 2012.

[24] A. Greco, G. Valenza, A. Lanata, G. Rota, and E. P. Scilingo, "Electrodermal activity in bipolar patients during affective elicitation," IEEE Journal of Biomedical and Health Informatics, vol. 18, no. 6 , pp. 1865-1873, 2014.

[25] A. Lanata, A. Greco, G. Valenza, and E. P. Scilingo, "A pattern recognition approach based on electrodermal response for pathological mood identification in bipolar disorders," 2014, pp. 3601-3605.

[26] A. Greco, G. Valenza, A. Lanata, G. Rota, and E. P. Scilingo, "Electrodermal activity in bipolar patients during affective elicitation," IEEE JOURNAL OF BIOMEDICAL AND HEALTH INFORMATICS, vol. 18 , no. 6 , pp. $1865-1873,2014$

[27] B. Maciel, L. Gallo, J. Marin Neto, and L. Martins, "Autonomic nervous control of the heart rate during isometric exercise in normal man," Pflügers Archiv European Journal of Physiology, vol. 408, no. 2 , pp. 173-177, 1987.

[28] R. Merletti and P. A. Parker, Electromyography: physiology, engineering, and non-invasive applications. John Wiley \& Sons, 2004, vol. 11.

[29] D. Farina and R. Merletti, "Comparison of algorithms for estimation of EMG variables during voluntary isometric contractions," Journal of Electromyography and Kinesiology, vol. 10, no. 5, pp. 337-349, 2000.

[30] A. Greco, G. Valenza, A. Lanata, E. P. Scilingo, and L. Citi, "Cvxeda: A convex optimization approach to electrodermal activity processing," IEEE Transactions on Biomedical Engineering, vol. 63, no. 4, pp. 797-804, 2016.

[31] M. Benedek and C. Kaernbach, "A continuous measure of phasic electrodermal activity," Journal of neuroscience methods, vol. 190, no. 1 , pp. 80-91, 2010 .

[32] H. F. Posada-Quintero, J. P. Florian, A. D. Orjuela-Cañón, T. AljamaCorrales, S. Charleston-Villalobos, and K. H. Chon, "Power spectral density analysis of electrodermal activity for sympathetic function assessment," Annals of biomedical engineering, vol. 44, no. 10, pp. 3124-3135, 2016.

[33] A. Greco, G. Valenza, L. Citi, and E. P. Scilingo, "Arousal and valence recognition of affective sounds based on electrodermal activity," IEEE Sensors Journal, vol. 17, no. 3, pp. 716-725, 2017.

[34] C. E. Martin, J. A. Shaver, D. F. Leon, M. E. Thompson, P. S. Reddy, and J. J. Leonard, "Autonomic mechanisms in hemodynamic responses to isometric exercise," Journal of Clinical Investigation, vol. 54, no. 1, p. 104, 1974

[35] P. H. Venables, S. Gartshore, and P. O'Riordan, "The function of skin conductance response recovery and rise time," Biological psychology, vol. 10, no. 1, pp. 1-6, 1980.

[36] R. Nikula, "Psychological correlates of nonspecific skin conductance responses," Psychophysiology, vol. 28, no. 1, pp. 86-90, 1991.

[37] A. Mundy-Castle and B. McKiever, "The psychophysiological significance of the galvanic skin response." Journal of Experimental Psychology, vol. 46, no. 1, p. 15, 1953. 